\title{
IN VITRO INCIDENCE OF ROOT CANAL BIFURCATION IN MANDIBULAR INCISORS BY RADIOVISIOGRAPHY
}

\author{
Simone Helena Gonçalves de OLIVEIRA ${ }^{1}$, Luiz César de MORAES ${ }^{2}$, Horácio FAIG-LEITE ${ }^{1}$, \\ Samira Esteves Afonso CAMARGO ${ }^{3}$, Carlos Henrique Ribeiro CAMARGO ${ }^{4}$
}

1- DDS, MSc, PhD, Professor, Department of Biosciences and Oral Diagnosis, Discipline of Anatomy, Dental School of São José dos Campos, São Paulo State University, São José dos Campos, SP, Brazil.

2- DDS, MSc, PhD, Professor, Department of Radiology, Dental School of São José dos Campos, São Paulo State University, São José dos Campos, SP, Brazil.

3- DDS, MSc, Doctorate Student in Biopathology, Dental School of São José dos Campos, São Paulo State University, São José dos Campos, SP, Brazil.

4- DDS, MSc, PhD, Professor, Department of Restorative Dentistry, Discipline of Endodontics, Dental School of São José dos Campos, São Paulo State University, São José dos Campos, SP, Brazil.

Corresponding address: Samira Esteves Afonso Camargo - Av. Dr. Jorge Zarur, 330 apto.56 - Jd. Apolo - 12243-081 - São José dos Campos, SP, Brazil. Phone: +55123911 4546 - e-mail: samiraafonso@uol.com.br

Received: March 6, 2008 - Accepted: October 09, 2008

\begin{abstract}
$O$

bjective: The aim of this study was to verify, in vivo and in vitro, the prevalence of root canal bifurcation in mandibular incisors by digital radiography. Material and Methods: Four hundred teeth were analyzed for the in vivo study. Digital radiographs were taken in an orthoradial direction from the mandibular incisor and canine regions. The digital radiographs of the canine region allowed visualizing the incisors in a distoradial direction using $20^{\circ}$ deviation. All individuals agreed to participate by signing an informed consent form. The in vitro study was conducted on 200 mandibular incisors positioned on a model, simulating the mandibular dental arch. Digital radiographs were taken from the mandibular incisors in both buccolingual and mesiodistal directions. Results: The digital radiography showed presence of bifurcation in $20 \%$ of teeth evaluated in vitro in the mesiodistal direction. In the buccolingual direction, $17.5 \%$ of teeth evaluated in vivo and $15 \%$ evaluated in vitro presented bifurcation or characteristics indicating bifurcation. Conclusions: Digital radiography associated with X-ray beam distally allowed detection of a larger number of cases of bifurcated root canals or characteristics of bifurcation.
\end{abstract}

Keywords: Digital radiography. Human. Mandibular incisors.

\section{INTRODUCTION}

The roots of mandibular incisors present mesial and distal longitudinal grooves that can lead to root canal bifurcation. Partial bifurcation occurs when these two canals join again and end in a single apical foramen. Total bifurcation is present when the two canals end in two apical foramina ${ }^{19}$.

Root canal bifurcation in mandibular incisors has great clinical importance during endodontic treatment. In teeth with partial bifurcation, obturation of one of the canals, generally the buccal one, seals the end of the other canal. On the other hand, in teeth with complete bifurcation, in which there are two independent apical foramina, poor or no filling of the lingual root canal may lead to failure of the endodontic therapy ${ }^{6,20}$. The presence of an untreated canal can result in persistence of symptoms and/or sign, such as pain and thickening of the periodontal ligament space ${ }^{3,4}$.

Several authors have studied the morphology of root canals of mandibular incisors and the results are very divergent in relation to the anatomic characteristics ${ }^{9,14,15,18}$. Some methods are available for investigation of internal anatomical variations in the daily clinical practice. Table 1 presents studies that employed radiographic techniques alone or combined with other methods. Authors who associated radiographic techniques to other methods found higher percentages of bifurcation ${ }^{2,7,16}$.

The aim of the study was to evaluate the prevalence of root canal bifurcation in mandibular incisors in an orthoradial direction by digital radiography. The study also compared the probability of detecting root canal bifurcation in vivo to its visualization with both buccolingual (BL) and mesiodistal (MD) projections in vitro.

\section{MATERIAL AND METHODS}

In this study, the prevalence of root canal bifurcation in mandibular incisors was verified in vivo and in vitro using a 
TABLE 1- Radiographic studies of the internal anatomy of mandibular incisors

\begin{tabular}{|c|c|c|c|}
\hline Authors and year & Method & Sample & $\%$ bifurcation \\
\hline Rankine-Wilson and Henry(1965) & $\begin{array}{c}\text { Injection of radiopaque material } \\
\text { and X-rays }\end{array}$ & 111 & $40.5 \%$ \\
\hline Benjamin and Dowson (1974) & Endodontic files and X-rays & 364 & $41.4 \%$ \\
\hline Hession (1977) & $\begin{array}{c}\text { Injection of radiopaque material } \\
\text { and X-rays }\end{array}$ & 13 & $61.5 \%$ \\
\hline Miyoshi, et al. (1977) & X-rays & 1.141 & $18.7 \%$ \\
\hline Bellizzi and Hartwell (1983) * & X-rays & 417 & $18.6 \%$ \\
\hline Neo and Chee (1990) * & X-rays & 154 & $1.3 \%$ \\
\hline Nattress and Martin (1991) & X-rays: BL and M-D & 455 & $13 \%$ \\
\hline Klein, et al.(1997) & $\mathrm{X}$-rays: $\mathrm{BL}$ and $\mathrm{MD}$ & 200 & $10 \%$ \\
\hline Oliveira, et al. (1999) & $\mathrm{X}$-rays: $\mathrm{BL}$ and $\mathrm{MD}$ & 2.115 & $10.3 \%$ \\
\hline
\end{tabular}

* in vivo studies; $\mathrm{BL}=$ buccolingual radiograph; $\mathrm{MD}=$ mesiodistal radiograph

digital radiographic system (Radiovisiography; RVG, Trophy, France). Four hundred teeth were analyzed for the in vivo study. Digital radiographs were taken in an orthoradial direction from the incisor and canine regions. The digital radiographs of the canine region allowed visualizing the incisors in a distoradial direction using $20^{\circ}$ deviation (Figure 1). All individuals agreed to participate by signing an informed consent form. The study protocol was reviewed and approved by the Institutional Review Board (Protocol n. 026/2000-PH/CEP).

The in vitro study was conducted on 200 mandibular incisors positioned on a model, simulating the mandibular dental arch. Digital radiographs were taken from the mandibular incisors in a BL direction (Figure 2). After examination, the same teeth were removed from the model and digital radiographs were taken in a MD direction (Figure $3)$.

The electronic sensor of the digital radiographic system,

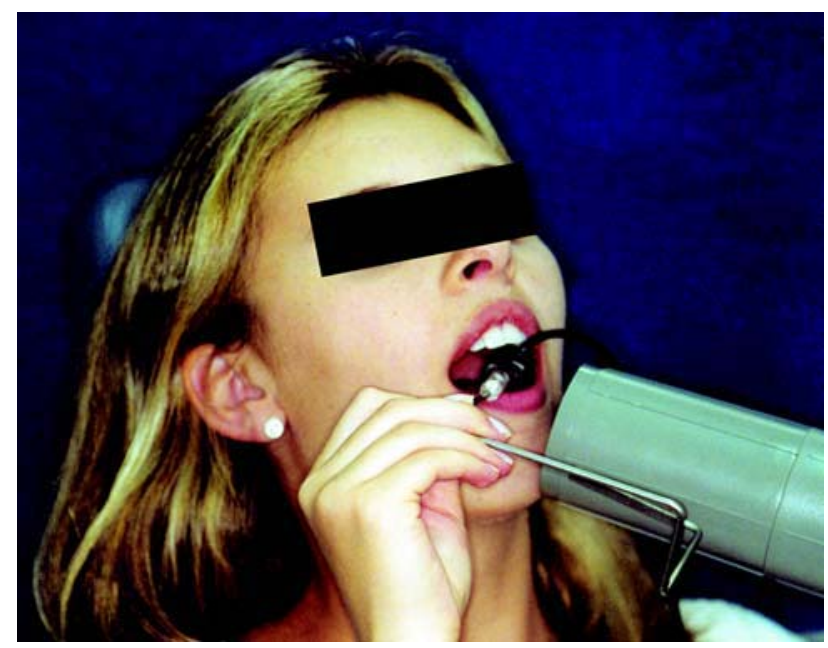

connected to the computer, generated images that were immediately seen on the monitor. Then, the digital radiographs were analyzed by a single examiner for the presence of a single root canal, bifurcated root canal, or root canal with characteristics indicating bifurcation These characteristics included decreased cervical-apical radiolucency and presence of radiolucent lines longitudinally on the root.

Data were submitted to statistical analysis by the chisquare test and the Yale's coefficient of association. Significance level was set at $5 \%$ for all analysis.

\section{RESULTS}

The in vivo study revealed $11 \%$ of root canal bifurcation canal and $6.5 \%$ of canals with characteristics that indicated the presence of bifurcation in a distoradial direction. These

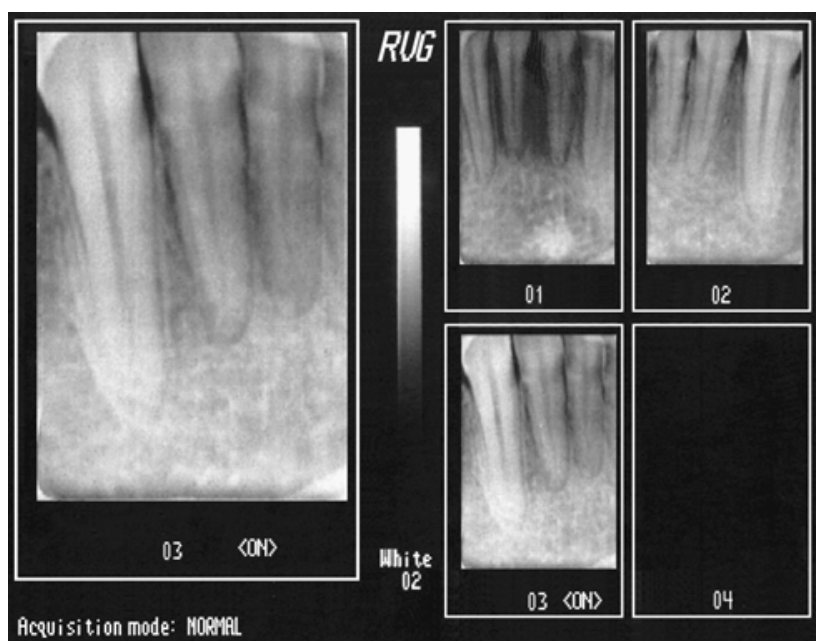

FIGURE 1- Examination (a) and images (b) in vivo with RVG 

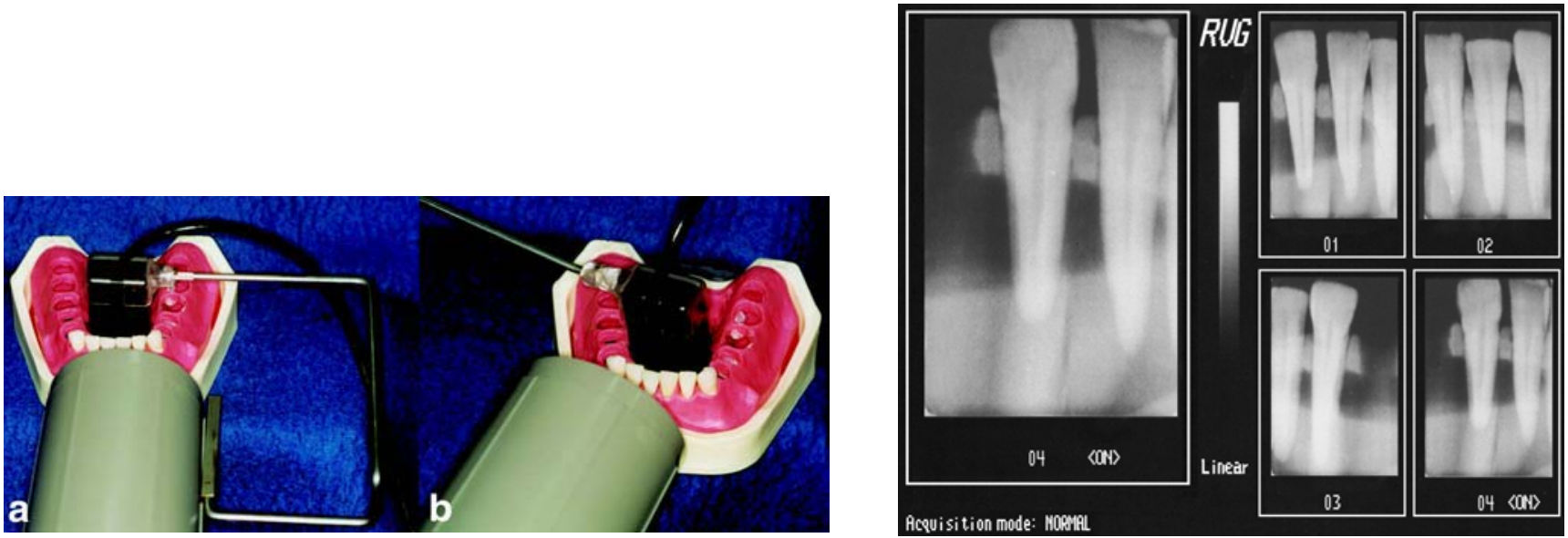

FIGURE 2- Examination ( $\mathrm{a}-\mathrm{b}$ ) and images (c) in vitro: in BL direction with RVG
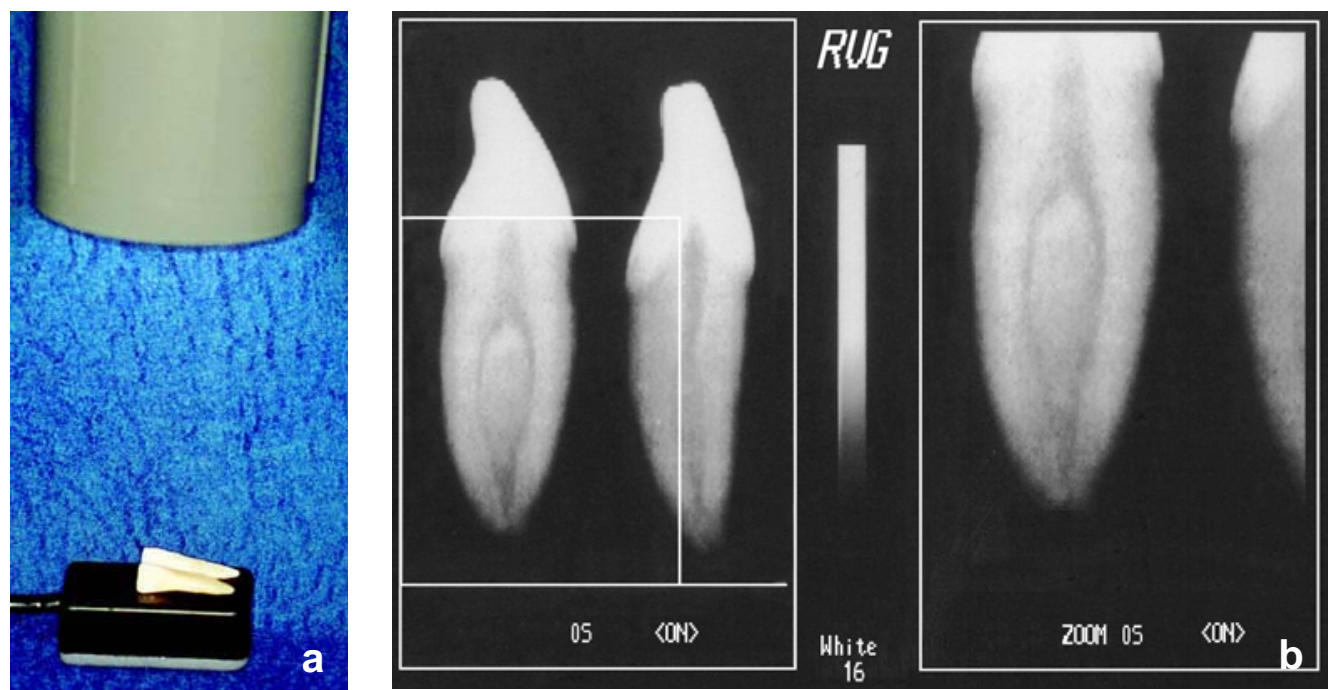

FIGURE 3- Examination (a) and images (b) in vitro in the MD direction with RVG

characteristics corresponded to loss of cervical-apical radiolucency and longitudinal radiolucent lines found laterally in the root due to the mesiodistal flattening. Therefore, in percent values, the in vivo study indicated that $82.5 \%$ of the mandibular incisors presented a single canal and $17.5 \%$ presented bifurcation or characteristics suggestive of bifurcation.

Figure 4 shows images in which root canal bifurcation was observed. Images in Figure 5 illustrate teeth with characteristics that indicated the presence of bifurcation. These images were captured during the in vivo examinations and represent loss of cervical-apical radiolucency and longitudinal radiolucent lines found laterally in the root.

The in vitro study with the digital radiography technique in a BL direction showed $15 \%$ of teeth with bifurcation or characteristics indicating bifurcation. In the MD direction, $20 \%$ of the teeth presented bifurcation or features suggestive of bifurcation.

Figure 6 presents images of teeth in both BL and MD directions.

The chi-square test was applied to the data collected from the in vitro study for comparison between digital radiographs in both $\mathrm{BL}$ and MD directions. This test was able to measure the discrepancy between two mutually exclusive qualitative variables (observation or not of root canal bifurcation or characteristics indicating bifurcation) and verified the independence or not between these variables. In the chi-square test, any result different from zero would indicate correlation between digital radiography in the BL and MD directions. A value of 52.41 was obtained.

However, the chi-square test cannot determine the degree of association: therefore, the Yale's coefficient of association was employed. A value of zero in this test indicates independence between samples; the maximum value of +1 indicates perfect positive association, and the value of -1 indicates perfect negative association. Our result for this test was +0.95 , indicating that there was perfect positive association between BL and MD directions.

Figure 7A presents graphically the sample distribution of the in vitro study. Seventy-seven percent of teeth presented single canal or absence of bifurcation in the BL and MD directions; $12 \%$ of teeth presented root canal bifurcation or 

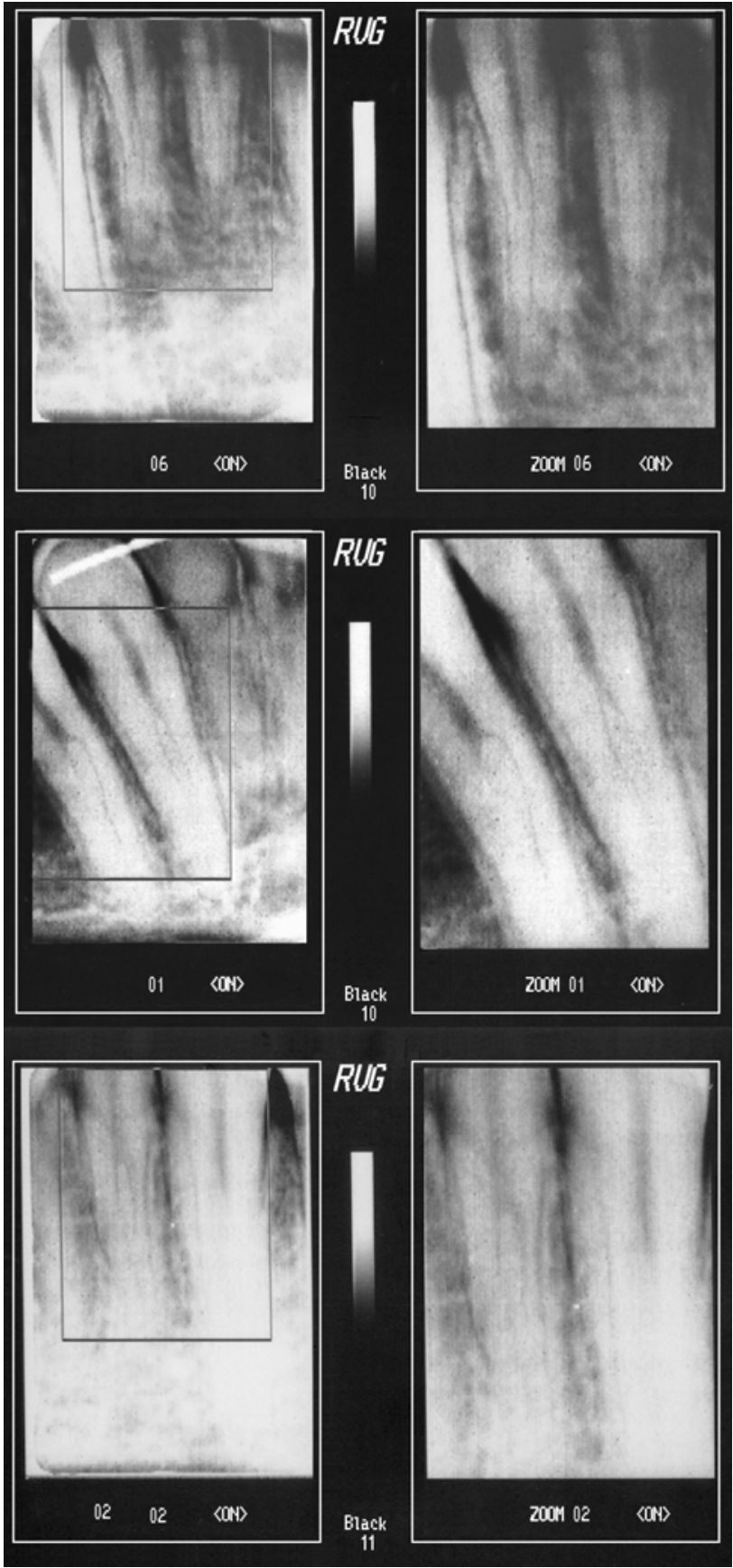

FIGURE 4- Images of root canal bifurcation in the in vivo study and magnification on the right side of the figure

characteristics indicating bifurcation in the BL and MD directions; $8 \%$ of teeth presented a single canal in the $\mathrm{BL}$ direction, yet root canal bifurcation in the MD direction; and $3 \%$ of teeth exhibited characteristics indicating bifurcation in the BL direction, yet presented a single canal when observed in the MD direction.

Statistical analysis was completed by observing the existence of correlation between the results from the in vivo and in vitro studies, by analysis of percentages of single and bifurcated canals (Figure 7B).
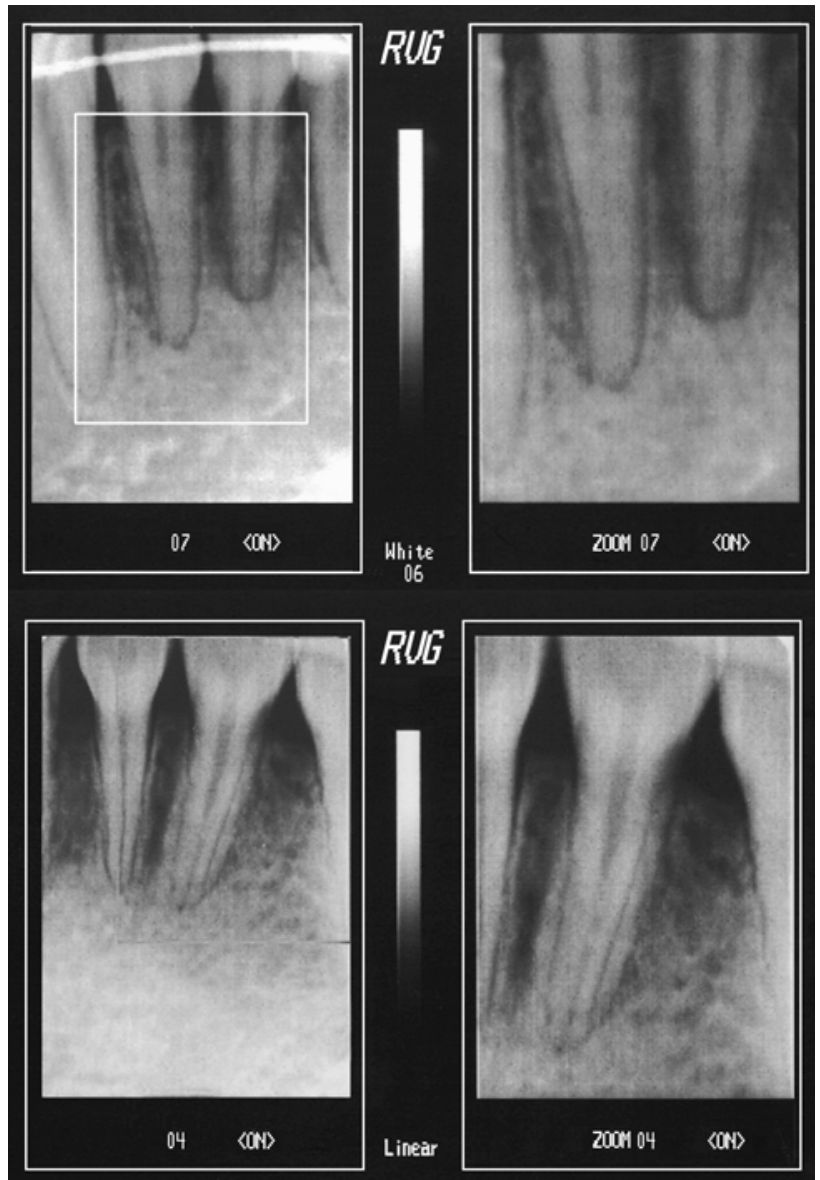

FIGURE 5- Images with characteristics indicating root canal bifurcation in the in vivo study and magnification on the right side of the figure
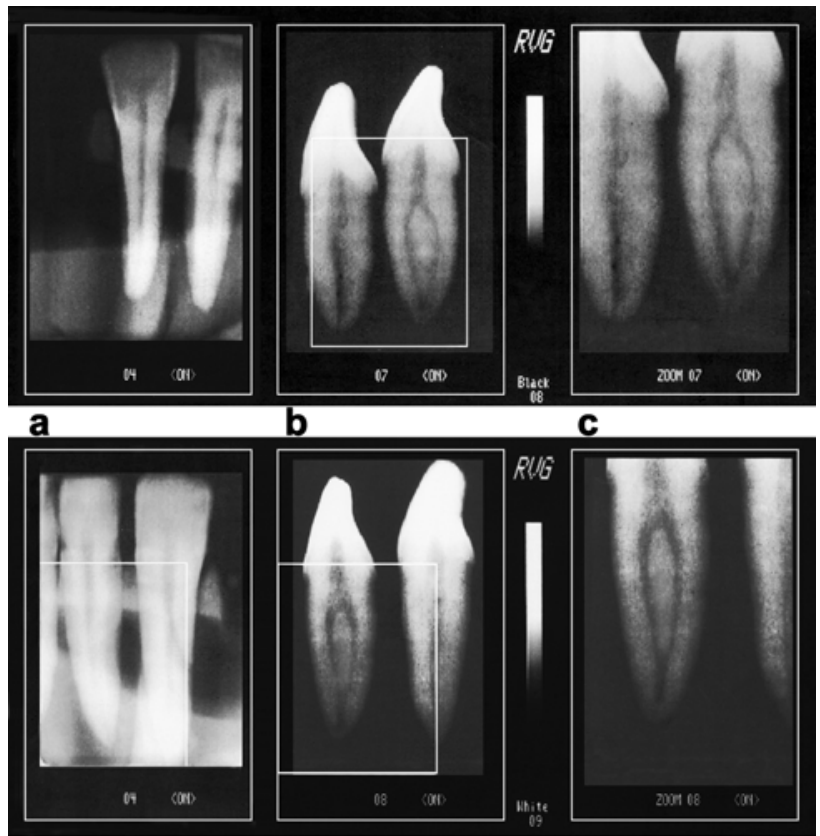

b

C

FIGURE 6- Images of in vitro study: a) orthoradial digital radiography of canine in the $\mathrm{BL}$ direction, b) digital radiograph in the $M D$ direction, $c$ ) magnification of digital radiograph in the $\mathrm{MD}$ direction 


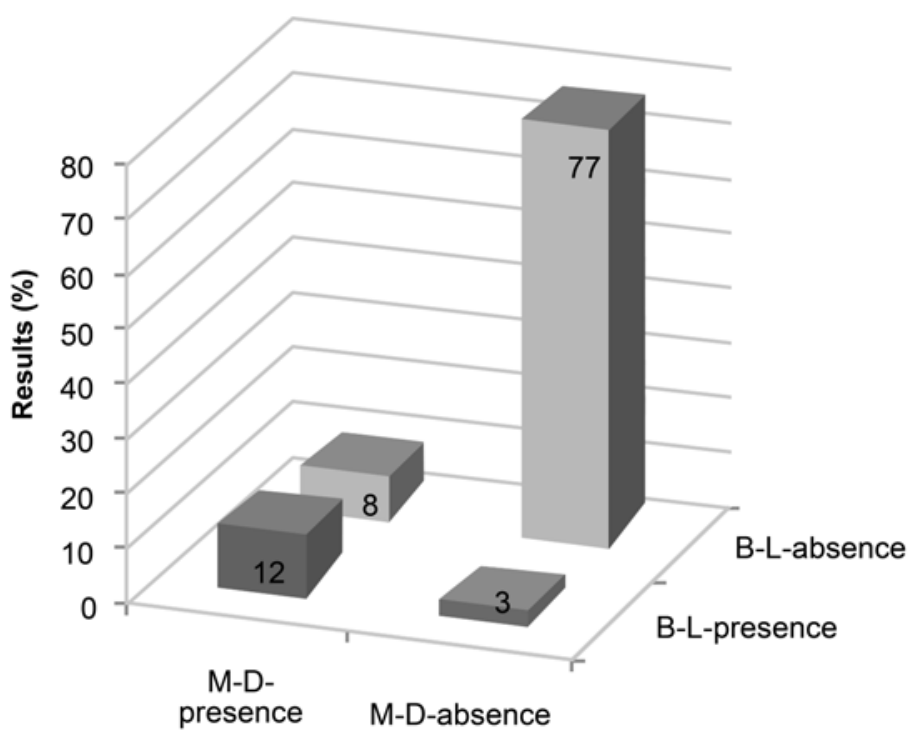

A

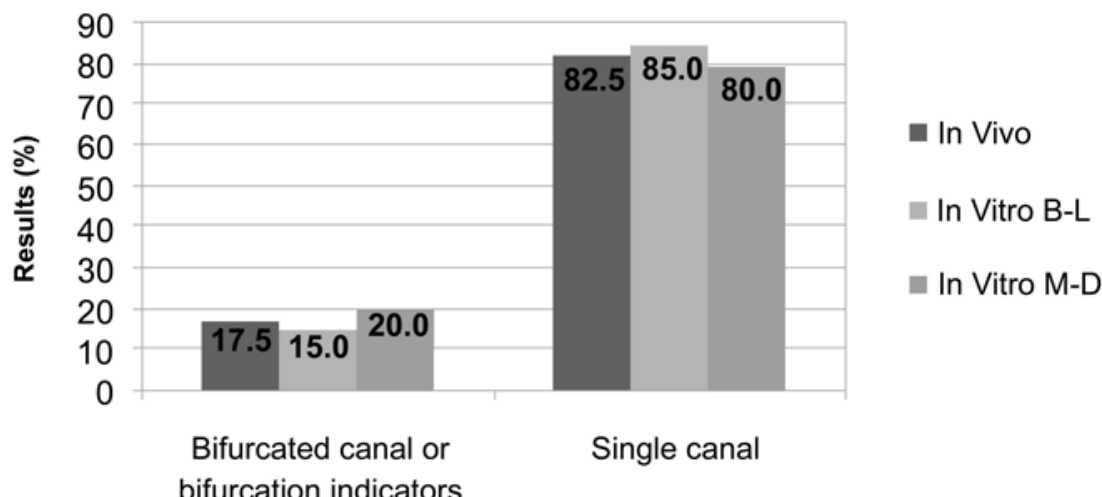

B

FIGURE 7- A- Graphic presentation demonstrating the comparison between the percent results of mandibular central and lateral incisors in the in vitro study. B- Graphic presentation demonstrating the percent correlation between in vitro and in vivo studies

\section{DISCUSSION}

The results of this in vivo study are similar to those found by Bellizzi and Hartwell ${ }^{1}$, who investigated 417 teeth and found $18.6 \%$ of bifurcated mandibular incisors. It must be emphasized that, in their study, those authors did not mention either the presence of bifurcation or the existence of characteristics suggestive of bifurcation, as mentioned in the present study.

Previous in vivo studies have reported percentages of bifurcation in mandibular incisors different from the present study ${ }^{9,15}$.

In the present study, sixteen teeth exhibited a single canal in the BL direction, though presenting root canal bifurcation when evaluated by digital radiography in the MD direction. Similar finding was reported by Nattress and Martin ${ }^{14}$, who investigated 455 teeth and found 17 teeth with a single canal when radiographed in the BL direction and bifurcated canals when analyzed in the MD direction. This result shows that, even with utilization of distoradial radiography, it was not possible to observe all cases of root canal bifurcation in mandibular incisors under clinical conditions. Thus, knowledge of root canal anatomy is necessary to avoid neglecting the presence of an additional canal.

The in vitro study, in which the teeth were analyzed by digital radiography in the MD direction, revealed a higher percentage of bifurcation (20\%) compared to the BL direction $(15 \%)$. These results were expected due to overlapping of root canal images in the BL direction. This was also observed by previous authors, who found higher number of bifurcation in incisors when these teeth are radiographed in the $\mathrm{MD}$ direction compared to $\mathrm{BL}$ direction ${ }^{14,18}$. Moreover, the in vitro study revealed that teeth with a single canal (40 teeth) in the BL direction also presented this characteristic in the MD direction. It has been verified that under a stereomicroscope, $15 \%$ of the teeth showed a bifurcated canal, $7.5 \%$ a lateral canal, and $25 \%$ accessory canals ${ }^{10}$.

In the present study, among the lateral incisors, 6 teeth 
presented root canal bifurcation or characteristics indicating bifurcation when analyzed in the BL direction. However, when the teeth were radiographed in the MD direction, this fact was not confirmed. In the literature, no authors that analyzed teeth in the BL and MD directions mentioned such occurrence ${ }^{12,13}$. This finding may be assigned to the fact that mandibular lateral incisors normally present root flattening in the MD direction, which can lead to appearance of a very marked longitudinal groove ${ }^{8,17}$.

Root canal bifurcation in mandibular incisors plays an important role in the endodontic treatment. In case of partial bifurcation, filling of one root canal, usually the buccal, will consequently seal the end of the other. On the other hand, this will not occur when there is total bifurcation with two apical foramina, since the lack of treatment of one canal will lead to failure of the endodontic therapy.

Some studies have reported that failure of treatment of mandibular incisors with two canals ending in a single apical foramen may occur due to poor or absent treatment of one of the canals ${ }^{5,11}$. Even though its end is sealed, the root canal may present lateral canals that can communicate with the periodontal tissues.

Clinicians, particularly endodontists, must be able to visualize all root canals of a tooth to fill them adequately ${ }^{6,20}$. For this purpose, professionals must have a good knowledge of the internal anatomy of teeth and also know the radiographic techniques and their variations, in order to have a perfect visualization of root canals. In this way, this study showed the importance to verify the presence of second canal in mandibular incisors for endodontic therapy and demonstrated that the diagnostic resources presented in this study may be helpful and effective.

\section{CONCLUSION}

The findings of the in vivo study (17.5\%) could be correlated with those of the in vitro study in both MD (20\%) and $\mathrm{BL}$ directions $(15 \%)$. The use of distoradial X-ray in vivo allowed detection of a good percentage of root canal bifurcation in mandibular incisors with the use of RVG.

\section{REFERENCES}

1 - Bellizzi R, Hartwell G. Clinical investigation of in vivo endodontically treated mandibular anterior teeth. J Endod. 1983;9(6):246-8.

2 - Benjamin KA, Dowson J. Incidence of two root canals in human mandibular incisor teeth. Oral Surg Oral Med Oral Pathol. 1974;38(1):1226.

3 - Fan B, Cheung GS, Fan M, Gutmann JL, Bian Z. C-shaped canal system in mandibular second molars: Part I-Anatomical features. J Endod. 2004;30(12):899-903.

4 - Fan W, Fan B, Gutmann JL, Cheung GS. Identification of C-shaped canal in mandibular second molars. Part I: radiographic and anatomical features revealed by intraradicular contrast medium. J Endod. 2007;33(7):806-10.
5 - Funato A, Funato H, Matsumoto K. Mandibular central incisors with two root canals. Endod Dent Traumatol. 1998;14(6):285-6.

6 - Gunday M, Sazak H, Garip Y. A comparative study of three different root canal curvature measurement techniques and measuring the canal access angle in curved canals. J Endod. 2005;31(11):796-8.

7 - Hession RW. Endodontic morphology. A radiographic analysis. Oral Surg Oral Med Oral Pathol. 1977;44 (4):610-20.

8 - Kabak YS, Abbott PV. Endodontic treatment of mandibular incisors with two root canals: report of two cases. Aust Endod J. 2007;33(1):2731 .

9 - Kaffe I, Kaufman A, Littner MM, Lazarson A. Radiographic study of the root canal system of mandibular anterior teeth. Int Endod J. $1985 ; 18(4): 253-9$

10 - Karagöz-Küçükay I. Root canal ramifications in mandibular incisors and efficacy of low temperature injection thermoplasticized gutta-percha filling. J Endod. 1994;20(5):236-40.

11 - Kartal N, Yanikoglu FC. Root canal morphology of mandibular incisors. J Endod. 1992;18(11):562-4

12 - Klein RM, Blake SA, Nattress BR, Hirschmann PN. Evaluation of $\mathrm{X}$-ray beam angulation for successful twin canal identification it mandibular incisors. Int Endod J. 1997;30(1):58-63.

13 - Miyoshi S, Fujiwara J, Tsuji Y, Nakata T, Yamamoto K. Bifurcated root canals and crown diameter. J Dent Res. 1977;56(11):1425.

14 - Nattress BR, Martin DM. Predictability of radiographic diagnosis of variations in root canal anatomy in mandibular incisors and premolar teeth. Int Endod J. 1991;24(2):58-62.

15 - Neo J, Chee LF. A retrospective clinical study of endodontically treated mandibular incisors in a selected Chinese population. Oral Surg Oral Med Oral Pathol. 1990;70(6):782-3.

16 - Rankine-Wilson RW, Henry P. The bifurcated root canal in lower anterior teeth. J Am Dent Assoc. 1965;70:1162-5.

17 - Shemesh H, van Soest G, Wu MK, van der Sluis LW, Wesselink PR. The ability of optical coherence tomography to characterize the root canals walls. J Endod. 2007;33(11):246-8.

18 - Sonntag D, Stachniss-Carp S, Stachniss C, Stachniss V. Determination of root canal curvatures before and after canal preparation (part II): A method based on numeric calculus. Aust Endod J. 2006;32(1):16-25.

19 - Walton RE. Endodontic radiographic techniques. Dent Radiogr Photogr. 1973;46(3):51-9.

20 - Yoshioka T, Villegas JC, Kobayashi C, Suda H. Radiographic evaluation of root canal multiplicity in mandibular first premolars. J Endod. 2004;30(2):73-4 